\title{
La responsabilité sociétale des professionnels à l'horizon du développement
}

\author{
Jeanne Simard et Marc-André Morency ${ }^{\mathrm{a}}$ \\ Laboratoire de recherche et d'intervention en gouvernance des organisations \\ Université du Québec à Chicoutimi
}

\section{INTRODUCTION}

La crise financière, politique et sociale qui sévit de par le monde depuis l'été 2007 n'a pas manqué de susciter, selon divers sondages nationaux et internationaux, un effritement de la confiance du public envers les institutions de régulation et les organisations privées.

La crise financière, politique et sociale qui sévit de par le monde depuis l'été 2007 n'a pas manqué de susciter, selon divers sondages nationaux et internationaux, un effritement de la confiance du public envers les institutions de régulation et les organisations privées. Au quotidien, les médias témoignent de vastes mouvements d'indignation et de contestation réclamant une meilleure gouvernance, une démocratie plus participative, une croissance pérenne, une diminution des inégalités, bref un véritable développement collectif. Ces sondages révèlent qu'il se produit une distance croissante entre les citoyens et une classe dirigeante dont font partie de nombreux professionnels ${ }^{\mathrm{b}}$. Au Québec, les ordres professionnels ont d'ailleurs été interpellés, d'une manière plutôt inconfortable, lors de commissions publiques d'enquête, comme la Commission Charbonneau visant l'industrie de la construction et la Commission Bastarache sur le processus de nomination des juges de la province.

Cette crise de confiance trouve aussi à s'alimenter dans les risques collectifs nouveaux qu'entraînent les avancées rapides de la science et de la technologie. Comment doit-on aborder des risques certes souvent difficiles à anticiper au temps présent, mais dont la concrétisation pourrait entrainer des effets graves et irréversibles pour la santé et l'environnement des générations présentes et futures?
Ces interrogations et remises en question qui touchent les plans politique, organisationnel et technologique, invitent, à l'occasion du $40^{\mathrm{e}}$ anniversaire du système professionnel québécois en 2014, à poursuivre la réflexion sur la responsabilité des 366000 professionnels au Québec à l'égard du développement des personnes, des organisations et des collectivités. Doivent-ils et peuvent-ils être encore des animateurs dans leurs organisations et communautés, contribuant à réduire les inégalités, les injustices? Sont-ils suffisamment outillés sur les plans éthique, théorique et pratique, pour aborder les défis collectifs et proposer des choix devant les nombreuses rationalités, les complexités qui s'offrent à notre regard? Quelle est l'ampleur de leurs responsabilités eu égard aux problématiques de gouvernance des sociétés et des organisations? Les professionnels font-ils toujours partie de la classe dirigeante ou ne sontils plus que des travailleurs salariés comme les autres, assujettis à leurs fonctions immédiates?

Après avoir rappelé la diversité des responsabilités du professionnel, nous verrons que les dimensions de l'action professionnelle responsable composent une pyramide de capacités comportant cinq paliers. Les professionnels se mobilisent à divers niveaux de prestation; ils participent dans le meilleur des cas à l'élaboration d'une nouvelle gouvernance démocratique du développement. Cela a pour implication que d'autres outils d'intervention que le contrôle et la surveillance doivent se trouver progressivement mobilisés afin d'ancrer la responsabilité sociétale au cœur de l'identité professionnelle. On devra en constater l'influence dans la formation initiale et continue, dans les dispositifs juridiques visant les ordres professionnels et les organisations publiques ou 
privées, dans les activités d'accompagnement en matière de délibération éthique. C'est dans ce plus large contexte que se réaliserait le renouvellement du professionnalisme. À défaut de quoi, le statut de «professionnel» pourrait se dissoudre dans la subordination commune aux salariés, dans un cadre global demeurant hors de contrôle.

\section{LES RESPONSABILITÉS DU PROFESSIONNEL : UN PHÉNOMÈNE MULTIDIMENSIONNEL}

\section{Comme des pelures d'oignon, les différents ordres de responsabilité se superposent en fonction de la complexité d'une tâche, du nombre d'intervenants impliqués. Un professionnel doit articuler dans sa pratique des responsabilités professionnelles, civiles, pénales (ou criminelles) et sociétales.}

Que signifie être responsable de ses actes? Le terme responsable, du latin respondere, signifie: «qui assume les conséquences de ses actes», «être imputable quant aux effets de ses actes ». La responsabilité se présente comme un concept central affectant toute prise de décision animée par un professionnel. Mais elle peut différer selon que l'environnement est déterminé, sans surprise, maitrisable, «probable » ou complexe et incertain, ce qui ne manque pas d'arriver fréquemment dans l'environnement actuel. Comme des pelures d'oignon, les différents ordres de responsabilité se superposent en fonction de la complexité d'une tâche, du nombre d'intervenants impliqués. Un professionnel doit articuler dans sa pratique des responsabilités professionnelles, civiles, pénales (ou criminelles) et sociétales.

À la base, le professionnel doit assumer les responsabilités liées à son statut, à son rôle; c'est ce que prévoient au Québec le Code des professions et divers règlements, dont les codes de déontologie. On y trouve énoncés des devoirs de confidentialité, de secret professionnel, d'intégrité, de non-conflit d'intérêts, d'honoraires raisonnables, de diligence, d'accessibilité des dossiers, de publicité, de bonne relation avec l'Ordre et les confrères, etc. Nos professionnels reçoivent de l'autorité étatique le privilège d'être les acteurs d'une mission de protection du public. Pris en défaut, le professionnel sera convoqué et diversement sanctionné par un comité de discipline de sa profession, qu'il se soit produit ou non des conséquences préjudiciables pour un client ou un patient.
Le professionnel peut également se voir imputer une responsabilité pénale et même criminelle en cas de conduite contraire à l'intérêt général «mettant en danger la stabilité de l'ordre public et entraînant donc un préjudice qui transcende l'individu pour atteindre le groupe social tout entier $»^{1}$. Ce type de responsabilité se traduit par la condamnation d'un tort grave causé aux biens, à l'environnement ou aux personnes, ainsi que des mesures d'indemnisation et de répression: il $\mathrm{y}$ aura, le cas échéant, obligation de restituer les biens dans l'état antérieur au méfait; dans les cas les plus graves, il pourra même y avoir une peine d'emprisonnement.

Le professionnel doit assumer en outre les devoirs et obligations prévus dans ses promesses contractuelles et dans différentes lois civiles ou usages. Advenant un manquement à ces obligations, le professionnel peut voir sa responsabilité civile engagée et, par conséquent, être tenu de réparer le dommage matériel, corporel ou moral subi par un tiers. La responsabilité civile traditionnelle suppose réunis trois ingrédients : un acte fautif, un dommage et un lien entre l'acte et le dommage. Au contraire de la responsabilité disciplinaire, «il n'y aura déclaration de faute professionnelle que si un geste ou une omission cause un dommage à autrui et si la preuve démontre que le professionnel n'a pas pris les moyens à sa disposition pour éviter ce dommage $»^{c}$.

Enfin, toutes ces responsabilités juridiques nous rappellent, chacune à leur façon, qu'il y a bien encore une dimension supplémentaire essentielle que doivent avoir à l'esprit les professionnels. Il s'agit de cet engagement beaucoup, plus large, de loyauté, de considération, qu'ils doivent assumer vis-à-vis la société, l'environnement et les parties prenantes. Le professeur-éthicien Georges Legault souligne l'importance, voire la centralité de cette dimension :

[...] une société se structure grâce aux professionnels et au travail professionnel, qui 
assure la qualité du vivre ensemble. Cette responsabilité sociale du professionnel devrait être au cœur de « l'identité professionnelle $»^{2}$.

Pour le professionnel, l'horizon de l'intervention sociétale parait large, complexe, de telle sorte qu'une telle obligation de responsabilité se définit plus difficilement ou moins concrètement, tellement les impacts peuvent être diversifiés. Est-ce qu'une action, un projet auront un impact sur l'environnement, la santé, le bien-être d'une collectivité? Est-ce que les générations futures seront capables de vivre avec un tel projet, une telle décision?
Le mouvement vers la «responsabilité sociétale » chez les professionnels représente un paradigme complexe qui s'oppose directement aux dérives et aux automatismes qu'engendrent les agents de marché laissés à eux-mêmes. Il invite à renouer avec la solidarité, la fraternité, avec un idéal démocratique, c'est-à-dire avec cette «capacité collective de définir un destin commun » plutôt que laisser champ libre aux intérêts individuels purement économiques. Le professionnel doit en ce cas de figure acquérir différentes capacités tout au long de sa carrière. C'est ce que précise la suite de notre analyse.

\section{LES DIFFÉRENTES FIGURES DU PROFESSIONNEL RESPONSABLE}

L'analyse sociologique doit fréquemment recourir à un instrument classificatoire, la typologie, afin de grouper les variantes d'une problématique. Nous proposons de distinguer cinq types pour représenter les différentes qualifications que devra ou pourra acquérir en carrière le professionnel. Ces types décrivent le cheminement progressif que parcourt un professionnel à compter de sa diplomation, lorsqu'il vise l'atteinte des plus hauts niveaux de qualification et de performance. Un professionnel peut élargir sans cesse le cercle de ses interventions au sein de sa société d'appartenance, jusqu'à pouvoir intervenir au plan stratégique, au sein des circuits d'influence.

\section{Schéma 1 - Les différentes figures du professionnel responsable}

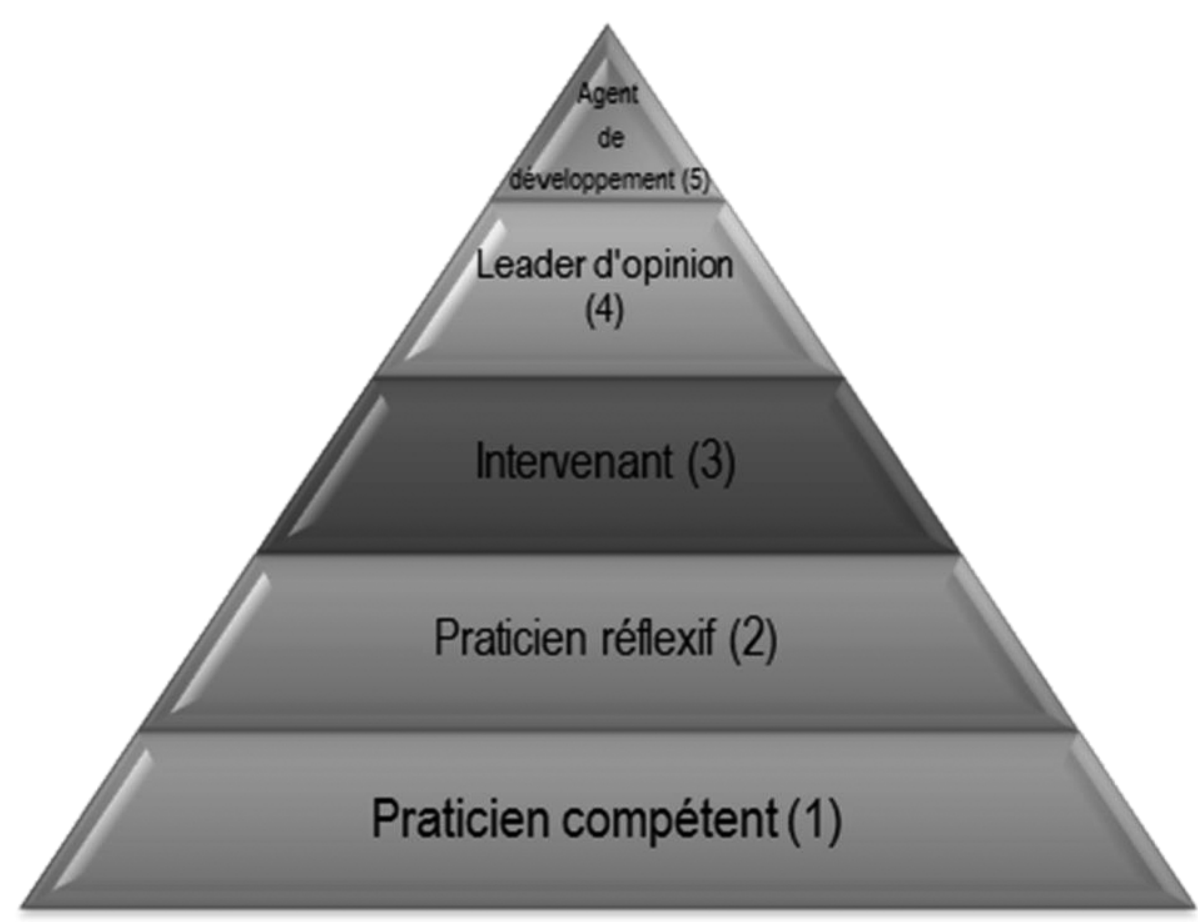




\section{Premier palier - le praticien compétent}

$\mathrm{Au}$ départ, le professionnel doit se présenter comme un praticien bien formé recevant, au terme d'examens approfondis de la formation initiale, une habilitation lui permettant de pratiquer, selon des standards bien définis reconnus comme tels par les pairs, un ensemble de prestations de services personnels, soit à titre partagé, soit à titre exclusif. Bien faire son travail à ce palier lui impose « d'être en mesure de donner une réponse techniquement performante à un problème; être compétent, c'est mobiliser le savoir-faire le plus approprié à une situation ${ }^{3}$. Comte-Sponville précise : «Être incompétent quand on a du pouvoir, c'est toujours faire preuve d'irresponsabilité. » ${ }^{4}$

Un professionnel se doit de maintenir cette qualification, que ce soit par voie de formation continue ou par l'étude personnelle des progrès dans la pratique, et ce, jusqu'au niveau de la spécialisation. Le rôle des ordres professionnels vise a minima le maintien de ce standard sur le plan de la protection des clients, en spécifiant les formations requises et en déployant des mesures a posteriori de vérification et de sanction. Tous les codes de déontologie professionnelle prévoient de nombreuses dispositions à cet effet. À titre d'exemple, le nouveau Code de déontologie des administrateurs agréés du Québec, adopté en 2014 , spécifie que «l'administrateur agréé doit exercer sa profession selon les normes généralement reconnues par les sciences et la pratique de l'administration. À cette fin, il doit veiller au perfectionnement de ses habiletés et à la mise à jour de ses connaissances ». De plus, «il doit, avant d'accepter de rendre des services professionnels, tenir compte des limites de ses aptitudes, de ses habiletés, de ses connaissances ainsi que des moyens dont il dispose ».

\section{Deuxième palier - le praticien réflexif}

Un professionnel qui avance dans sa carrière considère que sa pratique est plus qu'une simple application d'un savoir homologué, le standard d'une profession dans une situation donnée. Devant des attentes plus complexes de son ordre ou de la société, il tend à acquérir d'autres habiletés mobilisant des formes plus sophistiquées de réflexivité, une maîtrise langagière supérieure. C'est alors que l'éthique et le travail coopératif in situ prennent davantage de place, tout comme la capacité d'innover et d'évaluer les pratiques novatrices. Le professionnel se présente alors comme un «praticien réflexif ${ }^{5}$.

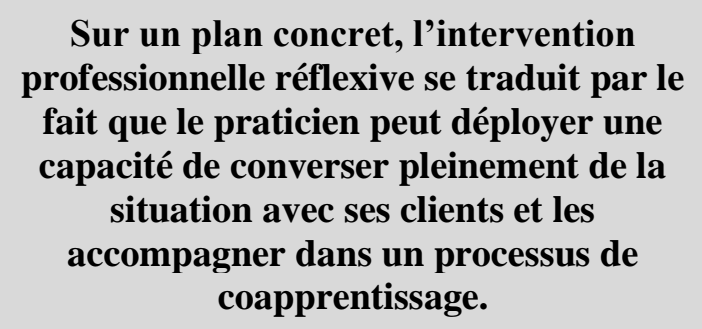

Sur un plan concret, l'intervention professionnelle réflexive se traduit par le fait que le praticien peut déployer une capacité de converser pleinement de la situation avec ses clients et les accompagner dans un processus de coapprentissage. La relation avec le client peut se modifier de façon à l'impliquer durablement dans la solution du problème à l'origine de la consultation.

Quand le médecin nomme le mal qui nous atteint, il construit un rapport symbolique à notre corps, il le transporte dans un univers de sens qui n'est pas celui du pur vécu immédiat: il le met en mots, lui permet d'accéder à la parole et à l'échange verbal. Alors le patient ne fait pas que subir sa douleur, il apprend à la connaître, à l'intégrer dans son identité personnelle, et, peut-être, à en faire un protagoniste de son action dans le monde ${ }^{6}$.

Le Barreau du Québec a ainsi constaté que la population apprécie ou valorise de plus en plus ce type de pratique, une situation qui oblige l'avocat à entamer une réflexion en profondeur sur ses méthodes de travail, à développer de nouveaux outils. Pour le Bâtonnier du Québec,

«C'est ce qu'on appelle l'empowerment. Les citoyens veulent faire partie de la solution. Ils n'acceptent plus, comme auparavant, l'autorité des avocats sans rien dire ni rien comprendre. [...] Nous ne pouvons plus dire comme auparavant: "Laisse-moi ton dossier, je m'en occupe". Les clients, désormais, veulent être associés à toutes les étapes du dossier, quand ils 
ne veulent pas tout simplement s'en occuper eux-mêmes » ${ }^{7}$.

On constate que la plupart des codes de déontologie des ordres professionnels obligent maintenant de développer cette étroite collaboration avec les clients, cette commune réflexion. À titre d'exemple, le Code de déontologie des administrateurs agréés mentionne qu' « en plus des avis et des conseils, l'administrateur agréé doit fournir à son client les explications nécessaires à la compréhension et à l'appréciation des services qu'il lui rend» ou encore qu'il « doit rendre compte à son client lorsque celui-ci le requiert ». C'est en fait une condition du maintien du lien de confiance entre le client et le professionnel.

Au-delà de la relation avec le client, le praticien réflexif commence à aborder des problématiques de développement. Il peut, par exemple, œuvrer à améliorer les performances énergétiques et sociales de son organisation, mettre en place une meilleure conciliation travail-famille, ou encore porter une attention toute particulière comme mentor au bien-être de jeunes professionnels se joignant à l'organisation. Enfin, dans un contexte plus global et comme le prescrivent la plupart des codes de déontologie, le praticien réflexif cherche à saisir les impacts «prévisibles » de ses actions, de ses projets et celles de ses pairs sur la société. "Compte tenu de son expertise, il [le professionnel] est, en effet, bien souvent le seul, avec ses pairs, à pouvoir juger convenablement des risques associés à son activité ${ }^{8}$. Il devient capable de prudence ${ }^{\mathrm{d}}$. La compréhension du professionnel réflexif doit alors se nourrir de considérations complémentaires, multidisciplinaires, de préoccupations sociétales.

\section{Troisième palier - l'intervenant}

Le praticien, comme intervenant, réfléchit aux causes structurelles à l'origine de problèmes se présentant dans le cadre de sa pratique. S'il considère par exemple la situation d'un groupe qui ne reçoit pas les soins et l'attention que requiert leur condition, il peut participer à un mouvement, à un groupe, voire inventer un instrument d'intervention'. Ces intervenants professionnels font la promotion d'approches de droit social, de santé publique, d'une saine alimentation, de l'exercice physique; ils font la critique de la malbouffe, promeuvent l'éducation populaire au moyen des médias, créent de nouvelles approches thérapeutiques à l'égard de clientèles de jeunes, en pédiatrie par exemple,

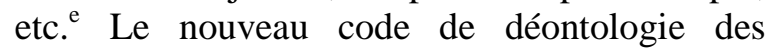
administrateurs agréés précise que «l'administrateur agréé doit favoriser les mesures d'éducation et $d$ 'information dans les domaines où il exerce sa profession. Il doit également favoriser toute mesure susceptible d'encourager la prise en compte de l'éthique dans les processus décisionnels ».

Ces interventions peuvent résulter d'initiatives personnelles ou de groupe, et le cas échéant, de plans dument établis par des autorités locales, régionales ou nationales. On se trouve à rejoindre de vastes opérations de développement sur le plan national ou international (lutte à la malaria, à la grippe aviaire, etc. $)^{\mathrm{f}}$. Les organisations professionnelles de type «sans frontières » en offrent des exemples multiples dans l'actualitég .

Ce qui vaut pour les professionnels s'applique mutatis mutandis aux ordres comme institutions publiques les représentant. Le président de l'Office des professions a rappelé que la mission de protection du public dont sont investis les ordres professionnels comporte une dimension sociétale. «Les ordres professionnels apportent un éclairage sur les enjeux de société ${ }^{10}$. On exhorte les ordres à s'ouvrir davantage à la communication publique, à ne pas confiner leur devoir de protection du public à la discipline et à la surveillance des membres. On insiste sur le rôle des ordres dans le maintien de la confiance comme élément central pour le système professionnel. En 2013, lors d'une allocution à l'occasion d'une journée de réflexion sur la gouvernance du Barreau du Québec, le président de l'Office déclare qu'il est de ceux « qui croient que la société a besoin que les institutions du système professionnel s'impliquent dans les débats publics et que les ordres ont avantage à jouer leur rôle sociétal pour contribuer à jalonner son évolution ${ }^{11}$. Il rappelle que la valeur centrale qui sous-tend la mission de protection 
du public confiée aux ordres professionnels est le bien commun et que, quarante ans après l'adoption du Code des professions, il est essentiel de réaffirmer et de se réapproprier cet objectif premier, de s'assurer que le public comprenne bien cet objectif, pour que la confiance envers le système s'accroisse. L'Office a profité de la révision en cours de la loicadre pour rappeler aux ordres de s'assurer que la «prévention » demeure au cœur de leurs actions, voire de la communication avec le public.

\section{Quatrième palier - le leader d'opinion}

Le professionnel peut intervenir, comme leader d'opinion, à ce titre, lorsqu'un changement dans les perceptions est nécessaire chez une portion importante de ses confrères ou de la population, par exemple, dans les cas de discrimination, de mal-perception de l'origine ou du traitement d'un problème sanitaire, etc. Un professionnel qui atteint la maturité dans son art jouira d'une certaine crédibilité supplémentaire du fait de sa position, de sa formation, de ses relations dans la communauté ${ }^{\mathrm{h}}$. S'il se trouve bien intégré dans cette communauté, voire reconnu comme un chef de file parmi ses pairs et qu'il s'exprime dans l'espace public, il aura la capacité de faire avancer des conceptions, des définitions de situation, d'en déplacer d'autres, dans la conscience collective.

Ce phénomène de diffusion accéléré des idées, des innovations, est d'autant plus accentué qu'il se trouve relayé par les médias de masse ou par les nouveaux médias ${ }^{12}$. On a pu observer et formaliser ces phénomènes accélérateurs de changements sociaux. Le sociologue James Coleman a pu démontrer que les médecins bien considérés par leurs collègues, et pionniers de nouvelles pratiques médicales dans leur milieu, amenaient des changements à se produire plus rapidement ${ }^{13}$. Il s'agit là d'un phénomène de portée générale qui s'applique mutatis mutandis aux autres catégories de professionnels. Rapidité et changement signifient que l'on assistera à des réalisations et à des coûts de mise en place bien moins onéreux. Les professionnels, dans ces questions de développement, font une différence soit en accélérant les changements, soit en étant impliqués dans le freinage que subira la collectivité du fait de leur résistance au changement.

\section{Cinquième palier - l'agent de développement}

La contribution intellectuelle du professionnel peut accroitre l'intelligence des problématiques collectives et contribuer à transformer les cadres institutionnels qui sont très précisément la substance du développement ${ }^{\mathrm{i}}$. Un tel professionnel devient capable de définir de nouvelles stratégies, de modifier les conditions de la gouvernance, l'équilibre des finances publiques, etc. Le développement qui se produit alors pourra stimuler une croissance durable, une expansion économique mieux orientée. Lorsque de telles contributions professionnelles se généralisent, dans un espace critique, et avec l'assentiment des citoyens, la démocratie se déploie alors concrètement, non pas comme une simple représentation théorique ou dogmatique de l'État, mais comme réalité vécue par cette collectivité. Afin de ne pas engendrer une polarisation de professionnels en conflit, qui à la limite neutraliserait les efforts, problème qui n'avait pas échappé à Schön ${ }^{14}$, il convient de former aux approches médiatrices ou collaboratives: on trouvera plus facilement des solutions à des problèmes de société qui divisent profondément l'opinion. Depuis les années 60, les régions du Québec ont pu profiter d'approches de concertation et de coopération, au sein de conseils régionaux, de sociétés de diversification économique, de conseils régionaux des élus, réunissant des représentants des divers corps sociaux, syndicaux, patronaux, municipaux, de toutes allégeances politiques, de milieux urbains et ruraux, etc. De nombreux professionnels et agents de développement y ont trouvé l'occasion de faire valoir et d'y accroitre leur capacité de travail interdisciplinaire.

En synthèse, ces figures plus complexes de responsabilité en matière de travail professionnel contribuent à améliorer la capacité d'autonomie des citoyens, font en sorte qu'ils soient plus en mesure de mener leur vie, de se responsabiliser, de s'émanciper, au lieu de devenir des mineurs ou des assistés, dépendants d'une «expertise » quelconque. Dans un pareil contexte, la figure du « grand leader omnipotent » perd sa place au profit d'une intelligence qui se veut un attribut de la 
collectivité, maitresse de sa destinée, comme cela a précédemment été défini ${ }^{15}$. Essentiellement, les professionnels sont appelés à devenir des animateurs de la prise de conscience et du débat public $^{\mathrm{k}}$. Les méthodes traditionnelles de prise en charge, voire d'infantilisation, perdent leur emprise

\section{CONCLUSION}

La crise en cours montre à nouveau qu'on ne peut plus, en conscience, considérer comme automatique le progrès social, comme s'il pouvait être une simple résultante $\mathrm{du}$ bon fonctionnement d'activités marchandes laissées à elles-mêmes. Il nous a paru utile, voire impératif, de jeter un regard attentif aux conditions et modèles permettant aux professionnels d'exercer de façon responsable et d'assumer pleinement leur rôle social dans un contexte de plus en plus global. Cette respon-sabilité a le caractère d'un appel, d'une mission qui implique un comportement à la fois transparent et éthiquement orienté à un véritable développement, au respect des lois en vigueur, en cohérence avec les normes internationales émergentes.

Sur un plan stratégique, force est de constater l'existence de diverses contraintes à la prise en compte de tout ce spectre de la responsabilité par les professionnels, ceux-ci n'ayant pas tous la même conscience des enjeux ni la même habileté dans la maîtrise éventuelle de toutes ces figures d'activité. Comme Luc Bégin le fait remarquer, «ce n'est pas tout le monde qui arrivera à adopter un point de vue suffisamment décentré de sa propre personne pour percevoir les impacts possibles de sa décision sur les autres personnes concernées; certains n'arriveront même pas à réaliser qu'il se pose en effet un problème d'ordre éthique dans cette situation ${ }^{16}$.

\footnotetext{
La formation initiale dispensée dans les universités devra se fonder sur la reliance, sur la coopération des disciplines, pour avoir prise sur les grands défis actuels en matière de développement. La formation continue des professionnels doit pouvoir conjuguer les savoirs de diverses disciplines pour réussir les mises à jour qui s'imposent.
}

dans de tels contextes. Le développement entraine ainsi une évolution des formes de démocratie. Développement et démocratie vont de pair: un professionnel sait reconnaître les lacunes existantes, s'indigner lui aussi et contribuer à l'essor collectif.

Le monde de l'éducation et de la formation professionnelle se trouve lui-même mis en question dans cette problématique. En matière de formation initiale, de formation continue, les silos disciplinaires, les formations professionnelles cloisonnées dans l'université, opposent encore un lourd obstacle aux diverses formes de transversalité, privant le futur professionnel d'une vision et d'une compréhension globales des problèmes individuels et collectifs. Les solutions et les approches innovantes réclamées par la critique aux fins de répondre à la complexité du réel peuvent difficilement y être incorporées. Comme le mentionne Edgar Morin, «tant que nous ne relions pas les connaissances selon les principes de la connaissance complexe, nous restons incapables de connaître le tissu commun des choses: nous ne voyons que les fils séparés d'une tapisserie $»{ }^{17}$. La formation initiale dispensée dans les universités devra se fonder sur la reliance, sur la coopération des disciplines, pour avoir prise sur les grands défis actuels en matière de développement. La formation continue des professionnels doit pouvoir conjuguer les savoirs de diverses disciplines pour réussir les mises à jour qui s'imposent. Un espace interprofessionnel de réflexion à cet égard devrait être institutionnalisé. Il pourrait comporter un dispositif de counselling en éthique et en intervention visant à aider les professionnels désirant contribuer plus activement à l'amélioration du vivre ensemble de la société et agir comme agents de changement. Ce counselling paraît d'autant plus nécessaire que, contraint par son triple statut, le professionnel, souvent salarié et toujours citoyen, peut trouver difficile de conjuguer ses obligations déontologiques et éthiques professionnelles, les obligations de loyauté envers son organisation définies par le contrat de travail, les règlements de régie interne et les soi-disant «codes d'éthique». Les exigences et les contrôles organisationnels peuvent être très pointilleux. L'observation des 
milieux de travail permet de repérer un certain nombre de problèmes, de tension et d'anxiété relativement à ces contraintes. Le professionnel salarié doit lutter pour conserver l'autonomie d'exécution et de jugement nécessaire à l'exercice $\mathrm{du}$ haut niveau de développement moral que réclament ses diverses responsabilités envers la société. Comme l'ont dénoncé Sumantra Ghoshal et Jeffrey Pfeffer, la «théorie de l'agence»n'a pas cessé de créer un environnement paranoïde dans les organisations ${ }^{18}$.

Face aux nombreuses exigences, le professionnel se trouve être un acteur social en transformation; idéalement, il assumera un comportement reconnaissant l'universalité du «vivre ensemble», la condition humaine elle-même. Cela l'amènera graduellement à assumer des situations de plus en plus complexes, requérant des approches multidisciplinaires, la collaboration interprofessionnelle, une capacité d'improvisation créatrice $^{19}$. Une telle prise de conscience conjuguera passion, courage, humilité, amour de la connaissance et du prochain. Cela répondra alors au vœu formulé dès 1966 par le président fondateur du Conseil interprofessionnel du Québec, Jules Deschênes, voulant que le professionnel s'intéresse à la chose publique et y contribue activement $^{20}$. Le professionnel a la responsabilité, pour emprunter les mots de Malarewicz, de « redonner une dimension humaine et solidaire à la vie sociale, comme une réponse possible face à l'anonymat de la globalisation ${ }^{21} \gg$.

\section{Face aux nombreuses exigences, le professionnel se trouve être un acteur social en transformation; idéalement, il assumera un comportement reconnaissant$$
\text { l'universalité du « vivre ensemble », la }
$$ condition humaine elle-même.}

\section{BIBLIOGRAPHIE}

${ }^{1}$ Baudouin, Jean-Louis et Patrice Deslauriers (2007), La responsabilité civile, $7^{\mathrm{e}}$ édition, Cowansville, Les Éditions Yvon Blais, p. 43.

${ }^{2}$ Legault, Georges (2004), «Identités et valeurs professionnelles », Colloque national, $30^{e}$ anniversaire $d u$ Code des professions et du système professionnel, organisé par le Conseil interprofessionnel du Québec, Montréal le 10 et 11 novembre 2004 [En ligne] http://agora.qc.ca/colloque/professions.nsf/Conferences/
Identite_et_valeurs_professionnelles (page consultée le 17 novembre 2011). On consultera aussi : Legault, Georges (2003), Crise d'identité professionnelle et professionnalisme, Presses de l'Université du Québec, Sainte-Foy.

${ }^{3}$ Castillo, Monique (2011) «Du professionnalisme à l'éthique professionnelle », Études, vol. 7, tome 415, p. $55-64$, p. 56

${ }^{4}$ Comte-Sponville, André (1998), Petit traité des grandes vertus, Paris, PUF/Perspectives Critiques.

CROP (2012), Confiance des Québécois envers les ordres professionnels et leurs professionnels, Québec.

${ }^{5}$ Schön, Donald Chris Argyris (1974), Theory in Practice: Increasing Professional Effectiveness, San Francisco, Jossey-Bass Publishers.

${ }^{6}$ Castillo, Monique (2011) «Du professionnalisme à l'éthique professionnelle », Études, vol. 7, tome 415, p. $55-64$, p. 61

${ }^{7}$ Nadeau, Jessica (2012), «Le bâtonnier et la société québécoise - Le Barreau entame une réflexion en profondeur ", Le Devoir.com, 6 octobre 2012.

${ }^{8}$ Bégin, Luc (2011), «La compétence éthique en contexte professionnel» dans L. Langlois (dir.), Le professionnalisme et l'éthique au travail, Québec, Presses de l'Université Laval, p. 105-122.

${ }^{9}$ Nélisse, Claude et Ricardo Zuniga (1997), L'intervention : les savoirs en action, Sherbrooke, GGC Éditions.

${ }^{10}$ Duhamel, Alain (2012), «Les professionnels veulent prendre leur place dans le débat public », Les Affaires, 21 janvier 2012. Dutrisac, Jean-Paul (2012), Notes pour l'allocation de monsieur Jean Paul Dutrisac, président de l'Office des professions du Québec, prononcée le 14 décembre 2012.

${ }^{11}$ Dutrisac, Jean-Paul (2013), Notes pour l'allocation de monsieur Jean Paul Dutrisac, président de l'Office des professions $d u$ Québec, prononcée le 20 décembre 2013, à l'occasion d'une journée de réflexion sur la gouvernance du Barreau du Québec.

${ }^{12}$ Katz, Elihu, Paul Felix Lazarsfeld (2008), Influence personnelle, Paris, Armand Colin : Institut National de l'Audiovisuel.

13 Coleman, James, Elihu Katz et Herbert Menzel (1970) [1957], « La diffusion des innovations chez les médecins » in Lazarsfeld, Boudon, Chazel (eds), L'analyse des processus sociaux, Paris, Mouton, 1970.

${ }^{14}$ Schön, Donald (1994), Le praticien réflexif. À la recherche du savoir caché dans l'agir professionnel, trad. par J. Heynemand et D. Gagnon, Montréal, Les Éditions Logiques.

${ }^{15}$ Paquet, Gilles (2011), La gouvernance collaborative, Montréal, Liber, p. 7.

${ }^{16}$ Bégin, Luc (2011), «La compétence éthique en contexte professionnel » dans L. Langlois (dir.), Le professionnalisme et l'éthique au travail, Québec, Presses de l’Université Laval, p. 105-122, p. 112. 
${ }^{17}$ Morin, Edgar (2011), La voie: pour l'avenir de l'humanité, Paris, Fayard, p. 152.

${ }^{18}$ Ghoshal, Sumantra (2005), «Bad management theories are destroying good management practices », Academy of Management Learning \& Education, vol. 4, no 1, p. 75-91.; Pfeffer, Jeffrey (2005), «Why do bad management theorie persist? A comment on Ghoshal ", Academy of Management Learning \& Education, vol. 4, no 1, p. 96-100.

${ }^{19}$ Le Boterf, Guy (2011), «Apprendre à agir et à interagir en professionnel compétent et responsable », Éducation permanente, vol. 3, no 188, p. 97-112.

${ }^{20}$ Deschênes, Jules (1966), «Les professionnels dans le Québec contemporain", Causerie donnée au Club Richelieu - Montréal, le 1er décembre 1966, p. 24.

${ }^{21}$ Malarewicz, Jacques-Antoine (2012), Systémique et entreprise. Mettre en cuvre une stratégie de changement, 3e édition, Paris, Pearson, collection Village Mondial.

\section{NOTES}

a Jeanne Simard, LL.D. est professeure-titulaire au Département des sciences économiques et administratives de 1'Université du Québec à Chicoutimi et responsable du Laboratoire de recherche et d'intervention en gouvernance des organisations (LARIGO); Marc-André Morency est sociologue et professeurretraité de l'Université du Québec à Chicoutimi.

${ }^{b}$ 2014. Edelman Trust Barometer/Annual Global Study. On consultera: http://www.edelman.com/insights/ intellectual-property/2014-edelman-trust-barometer/.

Edelman, firme de relations publiques mondiale, publie depuis 14 ans un baromètre de la confiance des consommateurs dans le monde envers quatre catégories d'acteurs institutionnels: les gouvernements, les entreprises, les médias et les ONG. Edelman a réalisé son dernier sondage en 2013-4 auprès de 33000 personnes résidant dans 27 pays dont le Canada).

${ }^{c}$ Ordre des dentistes du Québec (Syndic) c. Forget, 2001 QCTP 060.

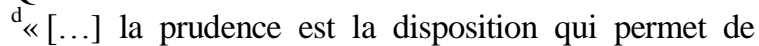
délibérer correctement sur ce qui est bon ou mauvais pour l'homme (non en soi, mais dans le monde tel qu'il est, non en général, mais dans telle ou telle situation), et d'agir, en conséquence, comme il convient » (ComteSponville, 1998 : 43).

eÀ titre d'exemple, trois infirmières ont mis sur pied un centre de prévention clinique pour la clientèle qui n'a pas accès à un médecin de famille. Elles ont reçu le Grand Prix Innovation clinique Banque Nationale 2012 pour ce projet qui mise sur la collaboration interprofessionnelle. On consultera : http:// www.oiiq.org/lordre/prix-et-distinctions/ concoursinnovation-clinique/recipiendaires-2012
${ }^{\mathrm{f}}$ De nombreux professionnels et intellectuels ont ainsi marqué l'histoire du Québec depuis la Révolution tranquille. Quelques exemples : Gilles Kègle, infirmier auxiliaire, a consacré toutes ses énergies à apporter des soins, du soutien et du réconfort aux personnes vivant dans la solitude et la pauvreté. Gilles Julien, pédiatre, a consacré sa carrière à promouvoir et développer la pédiatrie sociale pour le bien-être du plus grand nombre d'enfants possible.

${ }^{g}$ Plusieurs organismes canadiens et québécois se qualifient de «sans frontières». On y retrouve des dentistes, des médecins, des avocats, des acupuncteurs, des pharmaciens, des vétérinaires, des ingénieurs, des infirmières et infirmiers (Lachapelle, 2010).

${ }^{\mathrm{h}}$ Un exemple : Le docteur Réjean Thomas, médecin, a été président fondateur de Médecins du Monde, Canada et fondateur de la clinique L'actuel (à Montréal). Il est connu pour son dévouement et ses actions visant la prévention et le traitement des infections transmises sexuellement et du VIH-Sida.

${ }^{\mathrm{i}}$ Le développement est l'ensemble des transformations institutionnelles, psychosociales, mentales qui permettent une croissance durable, une diminution des inégalités, le maintien des cultures particulières, en symbiose avec l'environnement. En d'autres termes, le développement représente la montée en influence d'une intelligence collective et d'institutions, c'est-à-dire des manières de penser et de faire, par lesquelles les collectivités, les citoyens, contrôlent leur destinée en fonction de leurs intérêts de citoyens et de collectivités.

${ }^{\mathrm{j}}$ Un exemple : Louise Otis, présidente du Tribunal d'appel de l'Organisation internationale de la francophonie (OIF), s'est distinguée par la conception et l'implantation dans une cour d'appel, de l'un des premiers systèmes de conciliation judiciaire du monde. Elle a ensuite amélioré le système d'administration de la justice, au sein des Nations Unies, avec la création d'un système de médiation.

${ }^{\mathrm{k}}$ Un exemple de réussite : la Commission spéciale sur la question de mourir dans la dignité. On consultera : http://www.assnat.qc.ca/fr/travaux-

parlementaires/commissions/csmd-39-1/ 
Centre de recherche sur le développement territorial

\title{
Centre de recherche sur le développement territorial
}

\author{
UQAC - UQAR - UQAT - UQO - INRS-UCS - ÉNAP
}

Le Centre de recherche sur le développement territorial (CRDT) est un regroupement stratégique de chercheurs reconnu par le Fonds québécois de recherche sur la société et la culture.

Rassemblant près d'une cinquantaine de membres, dont une dizaine de l'extérieur du Canada, le CRDT est principalement rattaché à des institutions universitaires implantées au coeur même des régions du Québec.

Le CRDT met en oeuvre une programmation intégrée de recherche comparative sur le développement territorial :

AXE 1

Aménagement et gestion durables

iv du territoire et des ressources

AXE 2

Dynamiques économiques, production et proximité

AXE 3

Politiques publiques et gouvernance territoriale
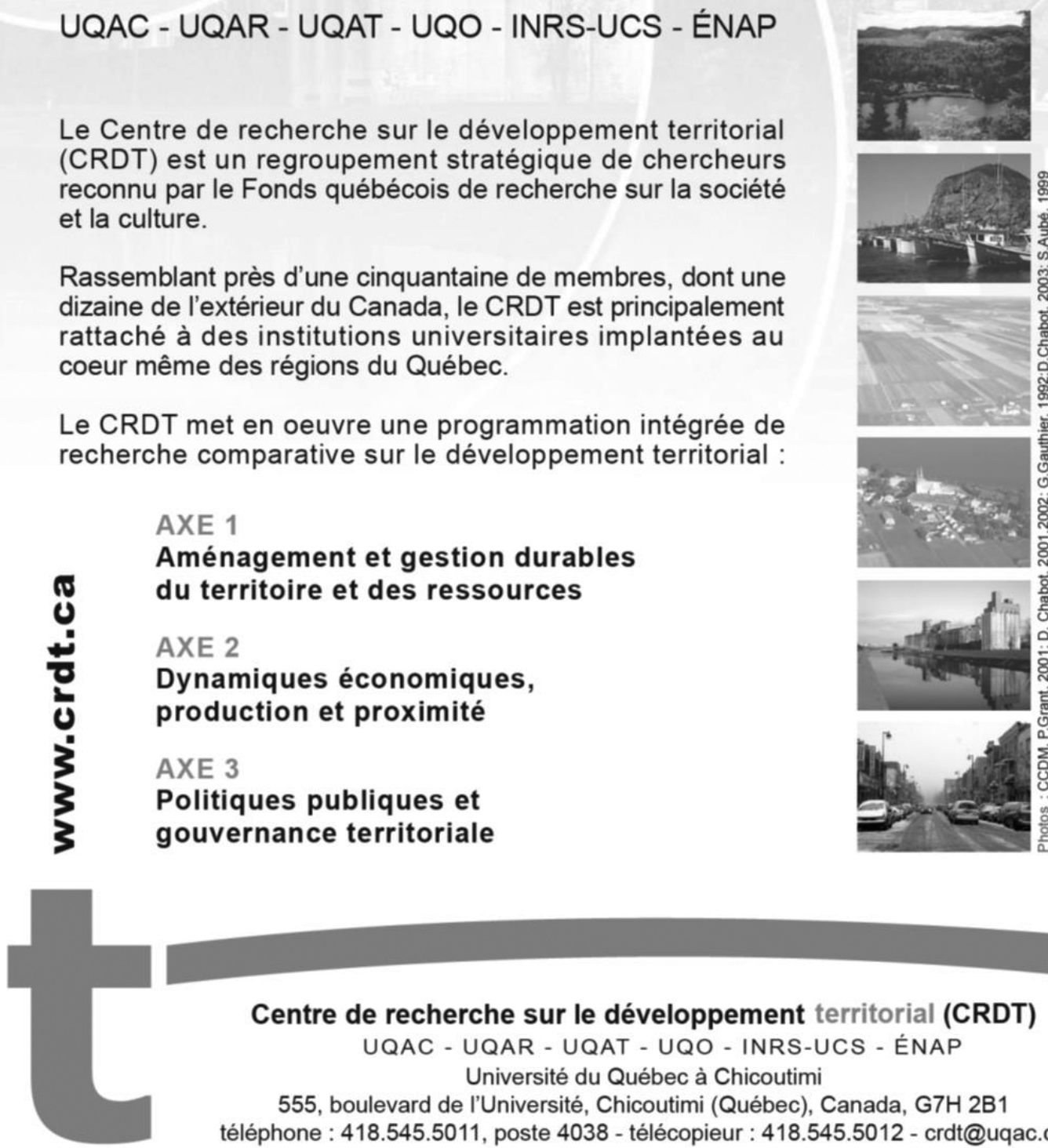

Centre de recherche sur le développement territorial (CRDT)

UQAC - UQAR - UQAT - UQO - INRS-UCS - ÉNAP

Université du Québec à Chicoutimi

555, boulevard de l'Université, Chicoutimi (Québec), Canada, G7H 2 B1

téléphone : 418.545 .5011 , poste 4038 - télécopieur : 418.545 .5012 - crdt@uqac.ca 